\title{
Otras vías de aprendizaje: el reconocimiento de la experiencia profesional en la universidad. El caso de la UNED
}

\section{Other ways of learning: the recognition of professional experience in the university. The case of the UNED}

\author{
Miriam GARCÍA-BLANCO, María José BAUTISTA-CERRO y \\ Marta RUIZ-CORBELLA \\ Universidad Nacional de Educación a Distancia
}

Recibido: Mayo 2014

Evaluado: Septiembre 2014

Aceptado: Abril 2015

\section{Resumen}

Nadie cuestiona que una fuente de aprendizaje es el propio ámbito de trabajo, en el que todo profesional resuelve las funciones y tareas a las que se enfrenta diariamente a partir de su formación y de su experiencia. Reconocer esta experiencia en las titulaciones de Grado es un tema aún no resuelto. Pero este reconocimiento exige un proceso de evaluación, apoyado en metodologías y herramientas fiables y válidas, a partir del cual se valide que una persona ha adquirido un determinado nivel de logro en unas competencias en el marco de una cualificación profesional determinada.

A raíz de los resultados obtenidos en 2 convocatorias dirigidas al reconocimiento de la experiencia profesional en el Grado en Educación Social impartido por la UNED, este articulo lleva a cabo un análisis de carácter descriptivo y en perspectiva comparada, entre ambas ediciones (2011-12 y 2012-13), con el fin de valorar la pertinencia de este proceso y la calidad de los resultados obtenidos. Con una muestra de 421 estudiantes hemos inferido la validez del procedimiento propuesto. Además, esta realidad nos muestra cuáles son las áreas de intervención más demandadas, los puntos fuertes y débiles de estos profesionales, las competencias más y menos consolidadas de acuerdo a la titulación que se imparte, etc. Disponer de estos datos, además de favorecer el desarrollo profesional de nuestros estudiantes, aporta una información clave para perfilar mejor las competencias específicas del título, trabajar de forma coordinada y colaborativa entre el mundo académico y el profesional, a la vez que responder a un derecho de nuestros estudiantes.

Palabras clave: competencia profesional, reconocimiento de competencias, acreditación de aprendizajes, educación superior, experiencia profesional 


\begin{abstract}
It is out of the question that a great source of learning takes place in the field of daily work, in which all professionals solve the functions and tasks that have to confront on a daily basis and based on their training and experience. Recognizing this experience in the Bachelor's degrees remains as an unresolved issue. But this recognition requires a process of evaluation, supported by reliable, valid and credible methodologies and tools, from which it can be recognized and certified that a professional has acquired a certain level of achievement in the competencies in the context of a particular professional qualification.

The results were obtained in 2 editions of the Social Education Degree imparted by UNED in which the recognition of professional experience was addressed. This article conducts an analysis of descriptive and comparative perspectives between the two editions (2011-12 and 2012-13) to assess the relevance of this process, the quality of the results and the response to this demand. Of the 199 applications in the first round and 222 in the second, it was confirmed that this was a study in which a significant number of students came from the same professional field, with proven experience of more than 9000 hours in an area of social intervention. This reality showed which areas are more demanded, the strengths and weaknesses of these professionals, as well as less consolidated competencies according to the degree, which was taught, etc. This information could enhance the professional development of our students, as it provides key information to sharpen specific competencies in these degrees, to work in a coordinated and collaborative relationship between academic and professional world, as well as to respond to a lawful right of our students.
\end{abstract}

Keywords: professional competence, competence recognition, validation of learning, higher education, professional experience

Cuando nos acercamos a analizar la repercusión que supone el paradigma de la educación a lo largo de la vida, comprobamos que muchos se limitan a hablar de la necesidad de formación en las diferentes etapas vitales, pero pocos abordan la verdadera revolución que implica este planteamiento: la desaparición de la hegemonía de la educación formal como espacio exclusivo de formación. Reconocer, con el mismo rango de acreditación, los aprendizajes adquiridos en diferentes escenarios supone un cambio radical para la planificación de los sistemas educativos, especialmente en la formación profesional y en la educación superior y su repercusión en la certificación de los títulos profesionales que se derivan de estos. Este hecho se está abriendo paso, poco a poco, no por convencimiento pedagógico, sino por la fuerza de los mercados que reclaman el logro del desarrollo económico y la empleabilidad, que no pueden perder o desperdiciar capital humano. De ahí que al potenciar el nivel formativo de sus profesionales, sea necesario fomentar vías de aprendizaje más flexibles, facilitar la transición entre trabajo y estudio y promover la validación de los aprendizajes no formales e informales (COM 2012a). Por ello, se hace necesario reconocer las competencias adquiridas, independientemente del escenario en que se hayan obtenido, con criterios de objetividad y rigor, de tal modo que pueden reconocerse con los mismos efectos académicos. Acercarnos a esta nueva demanda, impulsada también desde la Administración (European Commsion, 2014), es la intención de nuestra experiencia en la que se propone desarrollar, dentro de una 
práctica reflexiva, el procedimiento evaluativo que haga posible un reconocimiento de la experiencia profesional de calidad en las titulaciones de Grado de Educación.

\section{El reconocimiento de la experiencia profesional}

Entre los múltiples cambios que se derivan del planteamiento de la educación basada en competencias en la educación superior, en este artículo nos focalizamos en uno al que no se le está prestando la atención que se le debe: el reconocimiento de competencias de una titulación a partir de la experiencia profesional. Nadie cuestiona que una fuente de aprendizaje son los resultados de aprendizaje que se adquieren en los diferentes escenarios en los que cada individuo se desenvuelve y actúa. Ya no se trata de valorar únicamente aquellos que se logran en unas instituciones determinadas, sino de reconocer el dominio de competencias, independientemente de dónde se hayan adquirido (COM, 2012b). Y, hasta ahora, en las titulaciones que ofertan nuestras universidades aún no se ha abordado este reconocimiento, que exige la flexibilización de las diferentes vías de aprendizaje, si valoramos los logros alcanzados independientemente del proceso seguido (NUFFIC, 2012).

Entendemos las competencias como un constructo ligado a la acción, a la experiencia y al contexto socioprofesional, ya que estas entrañan no sólo un saber y un saber hacer, sino, fundamentalmente, la capacidad de saber movilizar los recursos necesarios en cada contexto y situación determinada (Tejada, 2012). Necesariamente este proceso de aprendizaje de competencias posee un carácter dinámico, lo que implica que puedan “(...) ser adquiridas a lo largo de toda la vida, constituyendo, por tanto, un factor capital de flexibilidad y de adaptación a la evolución de las funciones, los requerimientos y los empleos” (Tejada, 2012, p. 21). Este enfoque es el que justifica, en definitiva, el reconocimiento de diferentes escenarios para el aprendizaje y el que esté permanentemente llevándose a cabo. Es decir, no podemos seguir manteniendo la educación formal como única vía de formación, sino que se exige el reconocimiento, con las mismas garantías de rigor, de los aprendizajes generados en otros espacios y tiempos, (...) síntoma de que actualmente importa más lo que se aprende que las circunstancias de cómo, dónde, cuándo y con quién se aprende” (Sanz Fernández, 2005, p. 103). Reconocer nuevas fuentes de aprendizaje supone que la universidad reconozca otras vías de adquisición de competencias, entre las que "la experiencia laboral y profesional acreditada podrá ser también reconocida en forma de créditos que computarán a efectos de la obtención de un título oficial, siempre que dicha experiencia esté relacionada con las competencias inherentes a dicho título” (RD 861/2010, art. 6.2.)

Esto, a su vez, reclama mecanismos de validación rigurosos, transparentes y viables, que garanticen el reconocimiento de esas competencias adquiridas, ya que “(...) los mecanismos de validación permiten una mayor transparencia sobre las capacidades disponibles entre la mano de obra y ayudan a compaginar mejor las capacidades y la demanda laboral, promueven una mayor transferibilidad de las competencias entre empresas y sectores, y facilitan la movilidad en el mercado laboral europeo” (COM, 2012c, pp. 2-3). 
Entre las diferentes vías de reconocimiento de los aprendizajes que se están desprendiendo de esta realidad (Sobrado, 2003; Sanz Fernández, 2005; Medina y Sanz Fernández, 2009; NUFFIC, 2009, 2012; European Commission, 2014; etc.), en este artículo nos interesa centrar la atención en la acreditación de los aprendizajes por vía laboral o profesional en la educación superior. Tema poco conocido por la sociedad y, en particular, por la comunidad académica, y que aún despierta muchos recelos. No estamos ante una situación nueva, si tomamos como referencia la definición elaborada por la Organización Internacional del Trabajo (OIT) hace ya más de 3 décadas: "reconocimiento formal de las calificaciones ocupacionales de los trabajadores independientemente de la forma como hayan sido adquiridas” (Sanz Fernández, 2005, p. 101). En el que se exige que este reconocimiento del aprendizaje conlleve un proceso de evaluación, apoyado en metodologías y herramientas fiables, y válidas, a partir del cual se reconoce y acredita que una persona ha adquirido un determinado nivel de logro en determinadas competencias (COM, 2012c). De este modo, se equiparan las competencias adquiridas en espacios no formales y/o informales con las alcanzadas en una titulación, sin entrar en este momento en el debate del contenido y validez de cada uno de estos escenarios.

Además, no olvidemos que la formación en competencias está ligada siempre a la acción contextualizada (Tejada, 2011), por lo que no podemos reducir el proceso formativo de un profesional a un saber-hacer, sino, especialmente, en saber utilizar, aplicar en cada contexto y tiempo las más adecuadas (Tejada, 2012). Para ello es necesario conocer el qué, el por qué y el para qué de cada una de estas acciones, por lo que teoría y práctica se implican y reclaman necesariamente.

Estos procesos de reconocimiento de la experiencia adquirida requieren una evaluación de esas competencias ligadas a un perfil profesional, con el objetivo de certificar y acreditar el logro parcial o total de las mismas en un contexto determinado. Es decir, la acreditación del nivel de logro del desempeño laboral en una función concreta y en un ámbito de actuación específico. Ahora, si para la certificación de los aprendizajes formales tenemos ya un corpus de investigaciones y experiencias contrastadas que avalan su validez y rigor, no pasa lo mismo con las competencias adquiridas en otros escenarios, por lo que, a nivel de certificación formal, este proceso requiere aún de investigaciones y experiencias contrastadas. Sin duda, no pueden ser utilizadas las mismas herramientas que en la evaluación formal, dada su mayor complejidad, al tratarse de un aprendizaje contextual, que pretende una certificación formal. En este sentido, lo que se procura es “(...) abrir un proceso de evaluación para reconocer y certificar (eso es acreditar), en términos de enseñanza formal, las competencias que este trabajador ha podido adquirir a lo largo de su vida, mediante enseñanzas no formales (como la formación ocupacional y continua) o a través de procesos informales de educación (como la experiencia que ha adquirido a lo largo de su vida)” (Medina y Sanz Fernández, 2009, p. 168). Lo que nos conduce a diseñar un proceso de identificación, y valoración de evidencias de la competencia obtenidas por cualquier vía de aprendizaje (Retortillo, 2011).

En la política europea la validación del aprendizaje adquirido por vía no formal e informal aparece de forma sistemática a partir del 2001, cuando se asume el 
aprendizaje permanente, que conlleva la Declaración de los Principios Europeos Comunes para la Validación (2004), la creación del Europass (2004), como documento reconocido en el que se integra la formación y las cualificaciones y competencias adquiridas, el Marco Europeo de Cualificaciones para el Aprendizaje Permanente (2008), que recoge los niveles de cualificación a partir de resultados de aprendizaje, las directrices europeas para validar el aprendizaje no formal e informal (2009) y la creación de los sistemas de créditos basados en resultados de aprendizaje, como herramienta que facilita la movilidad y la validación de aprendizajes. Sin embargo, a pesar del gran consenso existente en dar visibilidad a las competencias adquiridas a partir de la experiencia profesional, se observa una gran disparidad en los procesos de validación de estos aprendizajes entre las diferentes naciones. Desde países que disponen de un sistema muy desarrollado (p.e. Francia, Reino Unido o Noruega), otros que lo han articulado a nivel jurídico, pero su implantación es aún muy lenta (p.e. España o Portugal) y, finalmente, aquellos que aún manifiestan una falta de confianza en esta propuesta (p.e. Eslovenia), ya sea por obstáculos culturales, económicos o de personas (FOREM, 2010; Werquin, 2010; COM, 2012c, 485; CEDEFOP, 2013a). Si bien en el nivel de Formación Profesional estos procesos de reconocimiento poseen ya un amplio bagaje de experiencias (FOREM, 2010; CEDEFOP, 2013b; Manzano, Martín Cuadrado y Ruiz-Corbella, 2012), no sucede lo mismo con la Educación Superior. Este nivel ha seguido las pautas de acción elaboradas por el CEDEFOP y en estos últimos años incluido en la agenda política (COM, 2012b; COM, 2012c), derivándose de ahí su inclusión en la política universitaria de todos los países como un factor para la empleabilidad y la movilidad. Si miramos a nuestra nación, estamos aún sumidos en el paso de la normativa a la realidad cotidiana en cada una de las instituciones universitarias.

\section{La validación de los aprendizajes por vía laboral en España}

En España, ante el alto índice de población activa con una cualificación profesional sin certificación oficial, se promovieron diferentes actuaciones enfocadas al reconocimiento, evaluación y acreditación de las competencias profesionales adquiridas mediante la experiencia laboral para el nivel de Formación Profesional. El marco clave para estas actuaciones se recogió en la Ley Orgánica 5/2002, de 19 de junio de las Cualificaciones y de la Formación Profesional, y a partir de ella se inició un trabajo sistemático que posibilitara este reconocimiento en todas las cualificaciones profesionales. El Instituto Nacional de las Cualificaciones (INCUAL), junto con las Comunidades Autónomas, desarrollaron el procedimiento y requisitos para la evaluación y acreditación de las competencias profesionales adquiridas mediante experiencia laboral (RD 1224/2009) en los niveles 1, 2 y 3 del total de cinco niveles de cualificación profesional (RD 1128/2003), por el que se regula el Catálogo Nacional de Cualificaciones Profesionales (Manzano, et al, 2012). Gracias a estas acciones se comenzó a dar respuesta a muchos ciudadanos que no podían acceder al ámbito laboral, al no estar en posesión de una certificación oficial, a la vez que se facilitó la inserción laboral de trabajadores sin esta acreditación. 
En la educación superior el proceso para posibilitar esta vía de reconocimiento siguió los mismos pasos: la Ley Orgánica 6/2001, de 21 de diciembre, de Universidad, modificada por la Ley Orgánica 4/2007, recoge por primera vez la validación a efectos académicos de la experiencia laboral o profesional. Sin embargo, no se desarrolló hasta 9 años más tarde, en el RD 861/2010 sobre la ampliación de los reconocimientos en los títulos de Grado, en el que se especifican los créditos objeto de este reconocimiento en un valor del $15 \%$ de la carga del título, pero no determina el procedimiento a seguir para su evaluación. De acuerdo a esta normativa cada universidad recoge esta vía de validación de los aprendizajes de forma muy diferenciada entre unas y otras, lo que ha limitado su efectividad.

Ante esta situación, es necesario que desde la universidad se diseñe un proceso que valore la experiencia profesional que presenta un estudiante de acuerdo con las competencias específicas establecidas para el Grado. Estamos, por tanto, ante un proceso en un doble nivel. Es preciso realizar un diseño que permita reconocer las competencias adquiridas por la vía de la experiencia profesional y, además sistematizar la traducción de esa competencia adquirida en número de créditos, materias y/o asignaturas. Todo ello deriva en dos preguntas clave: ¿Podemos conocer qué competencia/s ha adquirido una persona a través de la experiencia profesional? y si esto es factible, ¿cómo traducir este aprendizaje en créditos y asignatura/s?

Este proceso tiene una dificultad añadida, ya que normalmente la formación de una competencia responde a una o varias materias y, por lo tanto, a más de una asignatura. Los resultados de aprendizaje establecidos para cada competencia permiten valorar si un estudiante la posee o no y este indicio permite igualmente, a través de la realización laboral, comprobar si un profesional la ha adquirido. Por ello, el primer paso que exigen las preguntas planteadas será analizar las competencias específicas de una titulación, el nivel de logro que se ha establecido para ellas y las unidades de competencia que las integran, valorando si son susceptibles de ser aprendidas por diferentes vías.

En segundo lugar, es preciso diseñar el proceso y determinar y elaborar las herramientas en las que se va a apoyar el reconocimiento de la experiencia profesional, de tal forma que se garantice rigor, transparencia y calidad en esta validación. Y, en cada caso, identificar cual es el instrumento de evaluación que se ajusta más a este proceso valorativo: observación en el ámbito de trabajo, estudio de caso, entrevista, autoevaluación, etc.

En tercer lugar, especificar si la validación académica, de acuerdo a la especificidad del diseño de la titulación, se traduce en un número determinado de créditos y si se puede concretar en materias o asignaturas.

Todo esto nos lleva a afirmar que no se puede aportar un mismo diseño para todos los Grados, ya que cada título exige unos parámetros muy diferentes. E, incluso, la pertinencia de la validación de esta experiencia profesional presenta problemas muy diferenciados entre unos y otros, que van desde la imposibilidad de este reconocimiento en las titulaciones reguladas; la emergencia de la problemática del intrusismo profesional, lo que expone una situación sumamente compleja y con una 
dimensión ética relevante; la limitación de esta acreditación a asignaturas con una gran carga práctica, no pudiendo ser objeto de reconocimiento aquellas asignaturas clave en el desarrollo competencial de la titulación. No resulta fácil diferenciar en ocasiones unas de otras, a la vez que atender este derecho de todo estudiante. En este punto es relevante el trabajo conjunto que se debe llevar a cabo con los Colegios Profesionales a la hora de perfilar el contenido de los créditos objeto de reconocimiento, junto con la defensa de la profesión y de sus profesionales.

\section{El reconocimiento de la experiencia profesional en el Grado en Educación Social}

\section{Diseño de una experiencia piloto}

Desde el inicio de la implantación de la Diplomatura en Educación Social tanto los estudiantes, como el actual Consejo General de Colegios Profesionales de Educadoras y Educadores Sociales (CGCEES) reclamaron la posibilidad del reconocimiento de la experiencia profesional, respondiendo, así, a la amplia experiencia de muchos educadores que no disponían de una titulación universitaria. En ese momento, aunque se tenía constancia de esta opción en otros países, como es el caso francés, canadiense o australiano, el reconocimiento de la experiencia profesional aún no se contemplaba en nuestra normativa. Ante esta situación la única vía posible era que este reconocimiento formara parte de la asignatura de Practicum, como materia estrechamente vinculada a la experiencia práctica. Esta opción se desarrolló a lo largo de 8 cursos académicos (gráfico 1 y tabla 1), y en ella se valoró tanto la experiencia adquirida en escenarios profesionales como de voluntariado, reconociendo las horas de experiencia práctica en un centro de trabajo, aunque no se eximía de cursar los créditos teóricos de esta materia, centrada en la reflexión sobre su propia práctica.

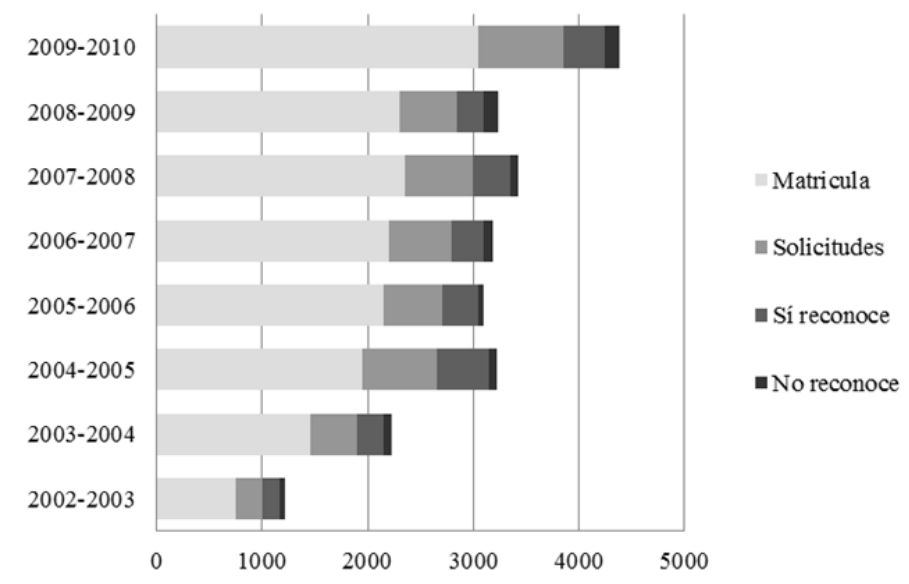

Gráfico 1. Datos de la experiencia de reconocimiento de la asignatura de Practicum en la Diplomatura en Educación Social. UNED. Cursos 2002-2010 


\begin{tabular}{|c|c|c|c|c|}
\hline Curso & Matricula & Solicitudes & Sí reconoce & No reconoce \\
\hline $2002-2003$ & 750 & 250 & 170 & 50 \\
\hline 2003-2004 & 1450 & 450 & 250 & 80 \\
\hline 2004-2005 & 1950 & 700 & 500 & 70 \\
\hline $2005-2006$ & 2150 & 550 & 350 & 50 \\
\hline 2006-2007 & 2200 & 600 & 300 & 90 \\
\hline 2007-2008 & 2350 & 650 & 350 & 70 \\
\hline 2008-2009 & 2300 & 550 & 250 & 130 \\
\hline 2009-2010 & 3050 & 800 & 400 & 130 \\
\hline
\end{tabular}

Tabla 1. Datos de la experiencia de reconocimiento de la asignatura de Practicum en la Diplomatura en Educación Social. UNED. Cursos 2002-2010

Esta experiencia supuso un beneficio para nuestros estudiantes y debemos resaltar la valiosa información que nos aportó al ponernos en contacto directo con la realidad del educador social, así como con las áreas de intervención más demandadas, sus necesidades formativas, sus puntos fuertes y débiles, etc. Además, se constató la necesidad de profundizar en el procedimiento de recogida de evidencias y ampliar las herramientas para la recogida de información y potenciar vías para favorecer la reflexión de los solicitantes sobre su práctica.

Una vez diseñado el título de Grado en Educación Social, desde la Facultad de Educación se propuso desarrollar las diferentes vías que la normativa facilitaba para el reconocimiento de créditos. Entre estas opciones figuraba la vía de la experiencia profesional. Para desarrollarla se contaba con la experiencia realizada en la Diplomatura, la participación de parte del equipo en el programa ACREDITA-T, promovido por varios Ministerios del Estado español de cara a favorecer la empleabilidad (Manzano, et al, 2012), el estudio de experiencias similares en el nivel universitario en universidades extranjeras, además del trabajo colaborativo con el CGCEES. En esta experiencia se acordó valorar exclusivamente las competencias adquiridas a partir de la experiencia profesional, dejando el sector del voluntariado, dada su singularidad y la necesidad de ser abordado de forma diferenciada, una vez se consolidara este procedimiento.

Algunas de las cuestiones fundamentales a tener en cuenta venían marcadas por la propia normativa que estableció el límite de créditos objeto de reconocimiento, que en el caso de este Grado se traduce en 36 ECTS. En cambio, el diseño del proceso, elaborado por un grupo de trabajo compuesto por profesores de la Facultad, fue fruto de la reflexión, el análisis de competencias y del aprendizaje contextualizado y el debate, que acordó que el procedimiento de acreditación girara en torno a tres fases 
consecutivas, especificadas en convocatoria pública, en las que el interesado aportara la siguiente documentación (figura 1):

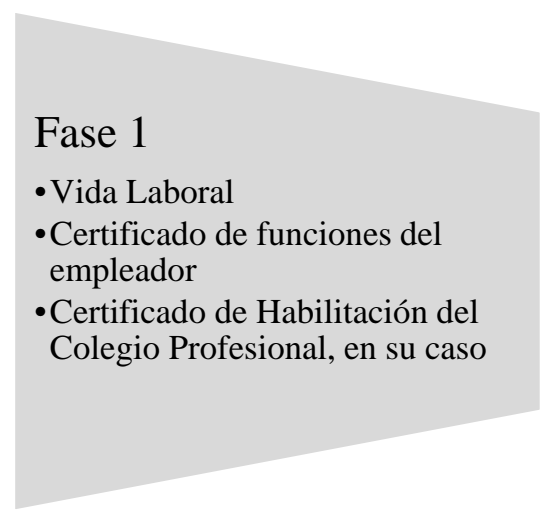

Fase 2

-Autoevaluación motivada (funciones, tareas y competencias)

- Solicitud motivada de asignaturas

Figura 1. Documentación requerida para el reconocimiento de la experiencia profesional. Fuente: elaboración propia

Todo el procedimiento y la documentación requerida era gestionada y valorada por los profesores del grupo de trabajo de la Facultad, que intervenía, tomaba decisiones y resolvía en cada una de las fases, mientras que las comunicaciones de las resoluciones se gestionaban a nivel administrativo, dado el carácter oficial del reconocimiento de créditos. Para iniciar este proceso, los interesados deben acreditar, al menos, 4.500 horas de trabajo (aproximadamente 3 años de trabajo), de las cuales al menos un 30\% deben haber sido desarrolladas en una misma función. Lógicamente, sólo se tiene en cuenta la actividad desarrollada en un área y función propia del educador social y en un nivel equiparable al titulado en educación superior. Se parte de la base de que las competencias específicas enunciadas en el Grado son competencias complejas que precisan de tiempo para su adquisición, pero además esto sólo se produce en contextos profesionales determinados. Para asegurar la actualidad de las mismas se valora la actividad profesional desempeñada en los últimos 8 años, de acuerdo a la lógica evolución de las profesiones (figura 2). 


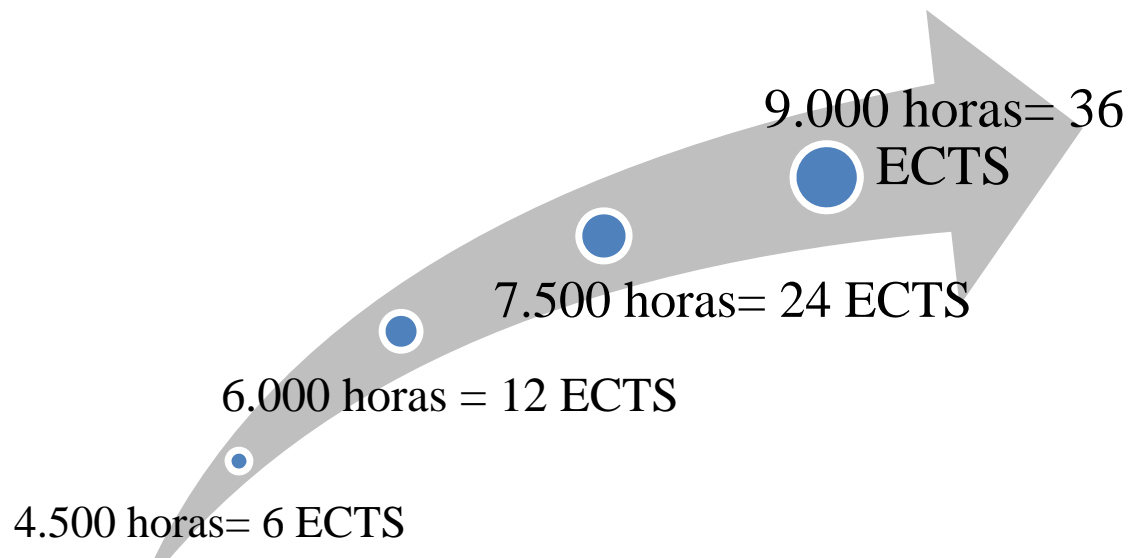

Figura 2. Distribución del número de horas y créditos ECTS para el reconocimiento de la experiencia profesional. Fuente: elaboración propia

Dada la relevancia de esta vía de reconocimiento, la solicitud de esta acreditación únicamente puede hacerse una sola vez a lo largo del título. De esta forma se incide en la necesaria reflexión, por parte del solicitante, sobre su propia práctica y en la preparación de las diferentes fases que componen la convocatoria. Esta capacidad de autoevaluación manifiesta, también, el grado de madurez formativa del estudiante, al tener que enfrentarse a elegir el mejor momento a lo largo de sus estudios para iniciar este proceso, y valorar las asignaturas que responden realmente a su experiencia profesional, ya que les permite justificar de manera más adecuada el nivel de logro alcanzado en las competencias de dichas asignaturas.

Otra decisión se centró en concretar el reconocimiento en asignaturas completas, por lo que se estableció una tabla de equivalencia entre número de horas de experiencia profesional y número de créditos ECTS.

Por último, se acordó ofertar una convocatoria anual con numerus clausus, en las primeras ediciones de 250 solicitudes, dada la alta exigencia de seguimiento individualizado que se requiere, ya que el trabajo de análisis de contenido de la documentación en las distintas fases es realizado por los profesores del grupo de trabajo. Estamos ante un proceso de reconocimiento que exige un importante tiempo de dedicación por parte de profesores y personal administrativo. Y, por otro lado, el interés por lograr que esta solicitud sea una petición reflexionada y motivada por parte de los estudiantes, hace necesario remarcar las diferencias respecto de otros procesos administrativos de reconocimiento, ya que tanto por su contenido como por la dificultad que entraña esta valoración, implica en el estudiante un nivel alto de implicación, participación y reflexión sobre su proceso formativo en diferentes contextos. 


\section{Metodología}

La metodología que llevamos a cabo para analizar el proceso de reconocimiento de la experiencia profesional es de carácter descriptivo y en perspectiva comparada entre las convocatorias que corresponden con los cursos 2011-12 y 2012-13. En el análisis se describen los resultados más destacados de la propuesta de reconocimiento de créditos adquiridos a través de la experiencia laboral de los estudiantes del Grado en Educación Social de la UNED.

En los 2 cursos en los que se ha desarrollado esta experiencia, ambos con una elevada matrícula de 7.988 y de 7.900 estudiantes respectivamente, se ha contado en cada convocatoria con unas solicitudes iniciales de 199 en la primera convocatoria y 222 en la segunda. Los estudiantes, para iniciar el trámite, deben cumplimentar un formulario en línea en el que reflejan los datos personales, a la vez que envían por correo postal la documentación original e imprescindible en esta primera fase: el Informe de Vida Laboral actualizado, el Certificado del empleador y el Certificado de Habilitación del Colegio Profesional, si es el caso. Resulta llamativo cómo en la primera edición el porcentaje de solicitudes que no llegan a formalizar el primer requisito, con respecto al número inicial de solicitudes recibidas, se eleva al 46,23\% (92), descendiendo este número de forma significativa al 21,62\% (48) en la segunda edición. Este dato se debe a los problemas que se encuentran a la hora de revisar su Hoja de Vida Laboral: número de horas reales cotizadas y tipo de contrato. O el problema derivado del certificado del empleador, donde deben aparecer reflejadas las funciones que ha desempeñado el solicitante.

\section{Análisis de los resultados de la experiencia}

El análisis de los resultados que aquí se expone refleja todos los datos con los que se ha trabajado en esta experiencia. Ello nos permite obtener información relevante sobre el propio proceso, su evolución y las posibilidades de mejora, de otro lado facilita el conocimiento de las ventajas e inconvenientes de un proceso que inevitablemente tienen que poner en práctica todas la universidades

La información sobre las horas y funciones desempeñadas se recoge a través del informe de vida laboral y el certificado del empleador, respectivamente. En ambas convocatorias el número de horas reconocidas supera las 9.000 horas (6 años), con unos porcentajes del 85\% en el curso 2011-12 y del 78\% en el 2012-13 (gráfico 2), lo que indica que la mayoría de los solicitantes cuentan con un amplio número de horas de trabajo desarrollando funciones como educadores/as sociales, por tanto, la puesta en marcha de esta experiencia responde a una demanda real de nuestros estudiantes. Será interesante contrastar este resultado dentro de 2 convocatorias, con el fin de valorar si esta realidad es una constante o si ya hemos dado respuesta a los profesionales sin titulación, por lo que debería reducirse significativamente esta demanda. 
Curso 2011-12

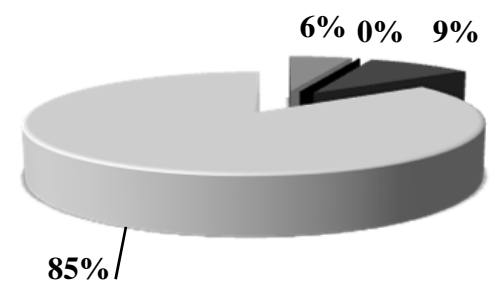

$\square 4500$ horas

घ6000 horas

$\square 7500$ horas $\square 9000$ horas
Curso 2012-13

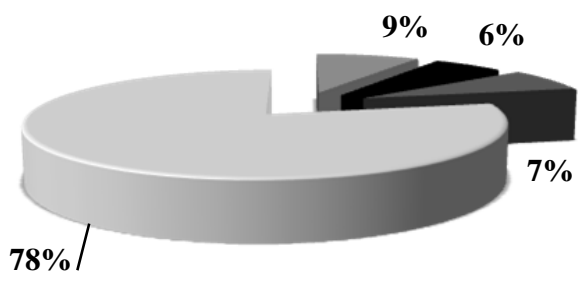

$\begin{array}{ll}\square 4500 \text { horas } & \square 6000 \text { horas } \\ \square 7500 \text { horas } & \square 9000 \text { horas }\end{array}$

Gráfico 2. Número de horas reconocidas. Cursos 2011-12 y 2012-13.

Fuente: elaboración propia

En el gráfico 3 se representan los porcentajes y el número de solicitudes que fueron aceptadas y desestimadas en la primera fase, en ambas convocatorias. Así, se observan porcentajes similares en las dos convocatorias, con un $77 \%$ de solicitudes aceptadas en la primera convocatoria y un $73 \%$ en la segunda. Los motivos por los que las restantes solicitudes son desestimadas se representan en el gráfico 4, donde la principal motivación es que la actividad que acreditan haber desempeñado los estudiantes, no se corresponde con un área específica de intervención socioeducativa; seguida por el motivo de no alcanzar las horas mínimas establecidas.

Curso 2011-12

25



Aceptadas

- Desestimadas
Curso 2012-13

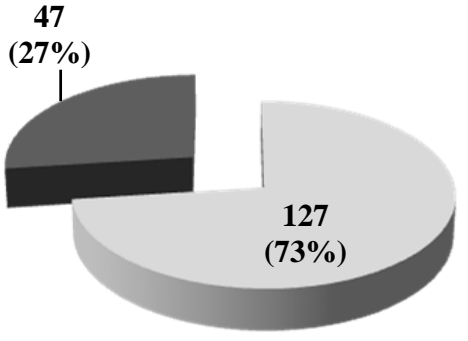

Aceptadas

- Desestimadas

Gráfico 3. Porcentaje y Número de solicitudes aceptadas y desestimadas en FASE I.

Cursos 2011-12 y 2012-13. Fuente: elaboración propia 


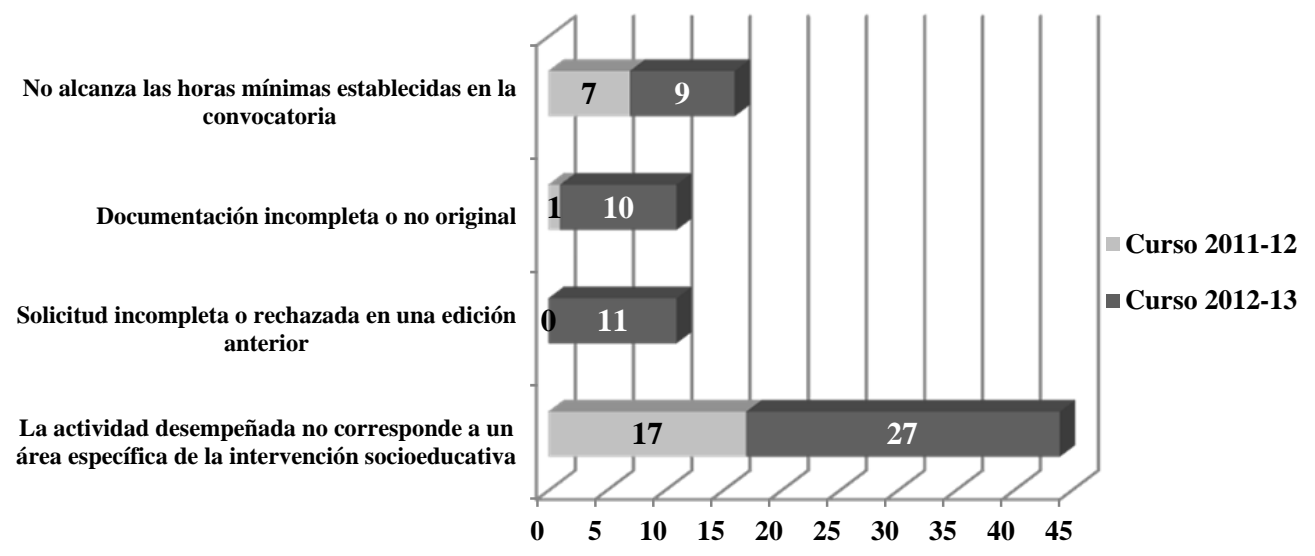

Gráfico 4. Motivos de denegación solicitudes desestimadas en FASE I.

Cursos 2011-12 y 2012-13*. Fuente: elaboración propia

* Conviene aclarar que los datos del número de solicitudes y los motivos de denegación pueden no coincidir, debido a que una misma solicitud ha sido desestimada por más de un motivo.

Con respecto a las principales áreas de intervención como educador/a social en las que desempeñan su labor profesional, la mayoría de los estudiantes que solicitan el reconocimiento de créditos llevan más de 6 años trabajando, tanto en el sector público como privado, en la atención a los menores, adolescentes y jóvenes en riesgo de exclusión social, pasando, en segundo lugar, a la atención a las familias (gráfico 5). Principalmente trabajan en hogares protegidos, residencias de menores, pisos tutelados, educadores de calle, etc. Este tipo de estudiante requiere la titulación para poder mantener su puesto de trabajo o para promocionar de categoría en el mismo, al estar contratados como `cuidadores', aunque desempeñan funciones de educador social.

Otras áreas de intervención socioeducativa representadas son la sociocultural, socioeducativa y social, relacionadas con programas de promoción social y desarrollo comunitario, de apoyo a la escolarización obligatoria y de lucha contra el absentismo escolar, de actividades extraescolares, animación sociocultural infantil, juvenil y de adultos, proyectos para la ocupación del tiempo libre... entre otros. Ahora, llama la atención la escasa presencia en áreas tan relevantes como la atención a la mujer, inmigrantes, discapacidad y mayores. 


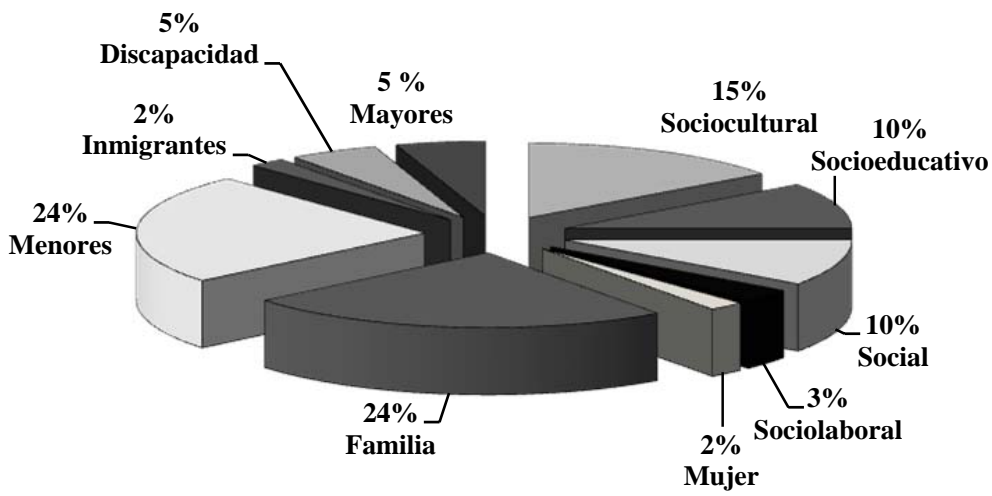

Gráfico 5. Áreas de intervención socioeducativa como Educador/a Social Fuente: elaboración propia

Una vez detectada el área de intervención, las horas y funciones acreditadas, los estudiantes cuyas solicitudes cumplen con todos los requisitos, pasan a la fase II del proceso, en la que adjuntan su currículum vitae actualizado y elaboran una autoevaluación motivada relacionando las competencias del título, con las funciones desempeñadas en su trabajo y la propuesta razonada de asignaturas objeto de reconocimiento, de acuerdo al análisis de competencias y funciones que ha desarrollado y analizado.

En esta fase II los resultados de las dos convocatorias son diferentes (gráfico 6). En la convocatoria del curso 2011-12, de las 83 solicitudes que pasaron la fase I tan sólo 53 (63,85\%) fueron aceptadas en fase II y, por tanto, sólo a ese $63,85 \%$ se le reconocieron asignaturas de la titulación, ya que 19 (22,89\%) estudiantes no enviaron la documentación solicitada en esta fase y 11 fueron desestimadas. Mientras que en la del curso 2012-13, de las 127 solicitudes aceptadas en fase I, 106 pasaron la fase II (el 83,46\%, 20 puntos porcentuales más que en la convocatoria anterior).

Curso 2011-12

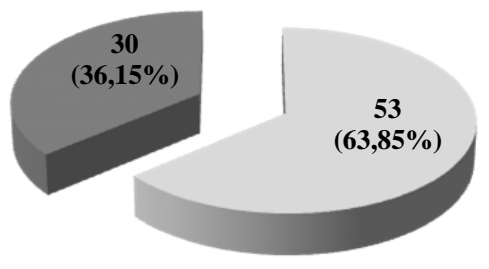

Aceptadas

Desestimadas
Curso 2012-13

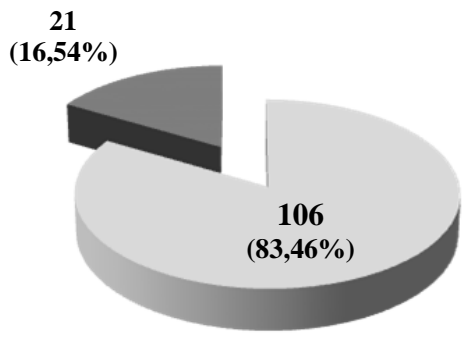

Aceptadas

- Desestimadas

Gráfico 6. Porcentaje y Número de solicitudes aceptadas y desestimadas en FASE II. Cursos 2011-12 y 2012-13. Fuente: elaboración propia 
Según estos datos, ¿cuáles son los motivos por los que las solicitudes de reconocimiento son desestimadas en la fase II del proceso? En el gráfico 7 se observan los principales motivos en cada una de las convocatorias, destacando en ambas la `no presentación de la documentación requerida en esta fase', lo que puede indicar que los estudiantes o bien no saben o no quieren realizar el ejercicio de autoanálisis y autoevaluación de las funciones de su experiencia profesional en relación con las competencias que han de adquirir en el Grado en Educación Social. Este aspecto es destacable porque al no presentar la documentación anulan todo el proceso y la oportunidad de obtener y/o solicitar de nuevo el reconocimiento, al arbitrarse la solicitud una sola vez a lo largo de la titulación. Para la resolución de dudas a la hora de desarrollar estos documentos pueden consultar a cualquiera de los miembros del equipo, opción utilizada por una minoría. Para futuras ediciones se está trabajando con el Centro de Orientación, Información y Empleo (COIE) de la universidad para ofrecer también asesoría desde este servicio.

En la convocatoria 2011-2012 la motivación referida a la `falta de concordancia entre las funciones desempeñadas, las competencias adquiridas y la propuesta de asignaturas para su reconocimiento' destaca sobre los restantes motivos. Esta cuestión muestra su bajo nivel de logro en un proceso de reflexión y autoevaluación de su propia práctica y desempeño como profesionales, a pesar de llevar muchas horas de trabajo como educador social.

En la convocatoria del curso 2012-2013 el principal motivo de rechazo de las solicitudes es la 'autoevaluación no personal', es decir, no elaborada de manera individual y personal por el estudiante. En segundo lugar, encontramos la `solicitud de asignaturas sin posibilidad de reconocimiento', como el Trabajo Fin de Grado que, de acuerdo a la normativa, no puede ser objeto de reconocimiento. Entendemos que estos resultados indican la escasa capacidad para identificar, seleccionar y analizar la información necesaria antes de tomar una decisión. Igualmente, encontramos como motivo de denegación la `falta de concordancia entre las funciones desempeñadas, las competencias y la propuesta de asignaturas' y la 'autoevaluación no motivada', al limitarse a indicar asignaturas sin avalarlas con una sólida argumentación. 




Gráfico 7. Motivos de denegación solicitudes desestimadas en FASE II.

Cursos 2011-12 y 2012-13. Fuente: elaboración propia

Como ya se ha indicado, el número máximo de horas para este reconocimiento de créditos es de 9.000, lo que implica más de 6 años de experiencia laboral, y que se traduce en 36 ECTS, que equivale a 6 asignaturas. En el gráfico 8 se refleja cómo en ambas convocatorias han sido reconocidas en mayor medida el número máximo de asignaturas posibles, lo que corresponde con los datos indicados en el gráfico 9 donde los estudiantes con más de 9.000 horas representaban el 85\% y el 78\%, en función de la convocatoria. 


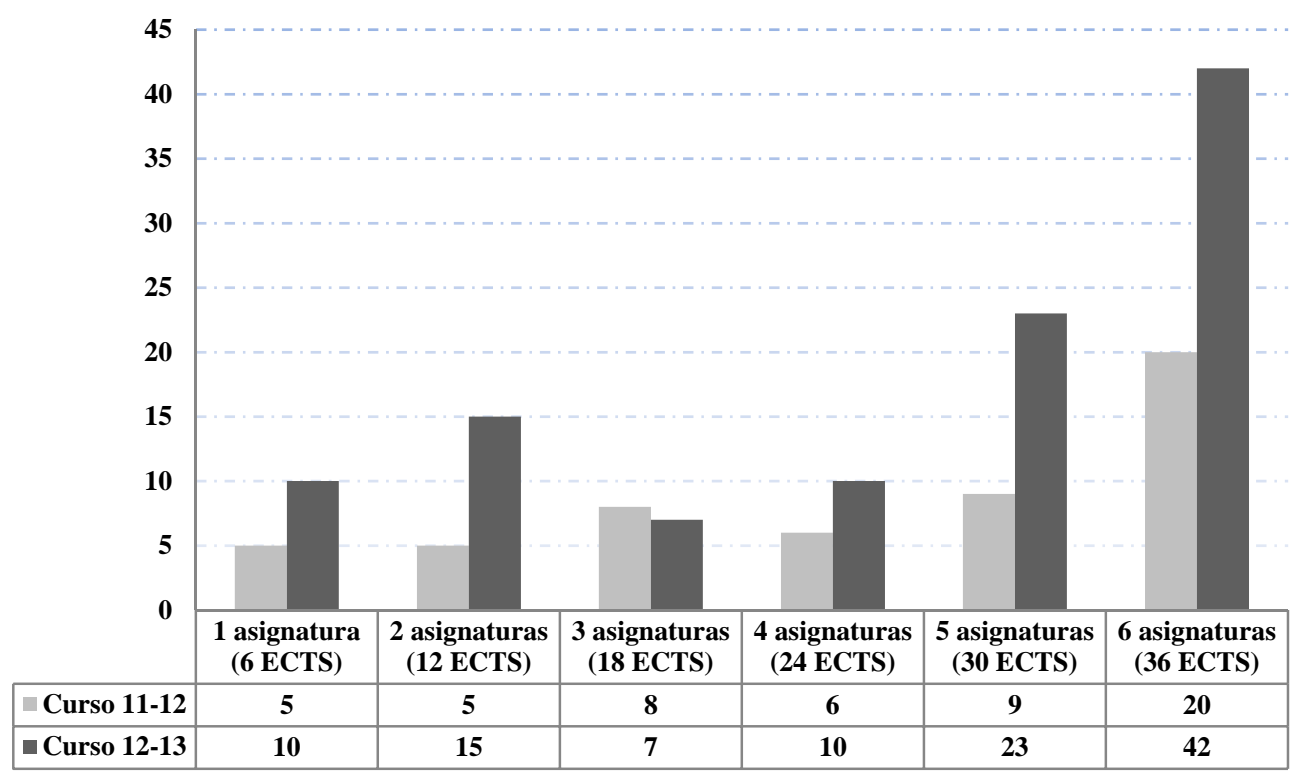

Gráfico 8. Número de asignaturas reconocidas. Cursos 2011-12 y 2012-13.

Fuente: elaboración propia

Las asignaturas que son reconocidas en primer término, son las relacionadas directamente con la materia de Prácticas Profesionales que, en este Grado se distribuyen en 5 asignaturas de 6 ECTS cada una (gráfico 9).

En el curso 2011-2012, además de las Prácticas Profesionales, se reconocieron asignaturas relacionadas con las áreas de intervención más usuales: 'Infancia, adolescencia y juventud en dificultad social'; 'Intervención socioeducativa para la prevención de la exclusión social', 'Asesoramiento y consulta en educación social'; junto con otras puntuales acordes con otros sectores.

En el curso 2012-2013 se sigue la misma tendencia de reconocimiento, pero no se focalizan en unas determinadas asignaturas al presentarse estudiantes procedentes de una mayor variedad de áreas de intervención socioeducativa. 


\section{Curso 2011-12}

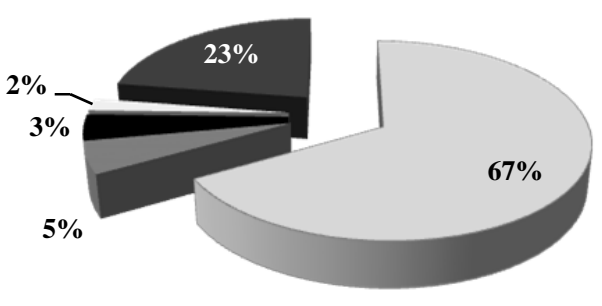

$\square$ Prácticas Profesionales

- Infancia, adolescencia y juventud en dificultad social

- Intervención socioeducativa para la prevención de la exclusión social

Asesoramiento y consulta en Educación Social

- Otras
Curso 2012-13

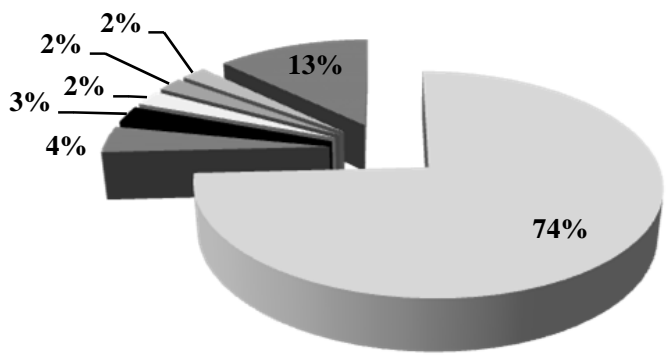

Prácticas Profesionales

- Infancia, adolescencia y juventud en dificultad social

- Intervención socioeducativa para la prevención de la exclusión social

Organización y gestión de instituciones y programas de intervención socioeducativa

- Mediación y orientación familiar

Evaluación de la intervención socioeducativa, agentes, ámbitos y proyectos

- Otras

Gráfico 9. Asignaturas reconocidas del Grado en Educación Social.

Cursos 2011-12 y 2012-13. Fuente: elaboración propia

A partir de la experiencia de la primera convocatoria, se vió la necesidad de completar en determinados casos la documentación aportada con una entrevista centrada en competencias, lo que se llevó a cabo en la segunda edición. En esta parte del proceso, cada entrevista fue desarrollada por 3 miembros del grupo. Esta versa sobre la documentación aportada en torno a la experiencia profesional, las competencias que motiva y las asignaturas solicitadas. De acuerdo a los resultados obtenidos a través de las entrevistas, se procede al reconocimiento, o no, de algunas de las asignaturas propuestas por el estudiante..A pesar de los inconvenientes que plantea la realización de las entrevistas, especialmente por el tiempo que conlleva, permiten contrastar con el estudiante la justificación tanto de las funciones desempeñadas laboralmente como de las competencias que dice haber adquirido a través de su trabajo como educador/a social. 


\section{Dificultades encontradas en la resolución de los expedientes durante el proceso de reconocimiento de la experiencia profesional}

Por parte del equipo de trabajo, la primera dificultad en sí misma fue elaborar un proceso (descrito anteriormente) que, con los recursos que cuenta nuestra Facultad (PDI y PAS), fuera capaz de ofrecer las suficientes garantías de calidad. De esta manera se intentó conjugar la aportación de documentación sobre la vida laboral de los solicitantes con la elaboración personal de documentos que reflejaran las competencias y la madurez propia de un profesional. Esta cuestión, que resultaba novedosa respecto al anterior proceso llevado a cabo en nuestra Facultad, fue la que presentó un mayor nivel de dificultad para los solicitantes.

Por otro lado, otra dificultad añadida fue encontrar unos criterios consensuados y fácilmente identificables a la hora de valorar las solicitudes, de manera que el proceso fuera equitativo a pesar de la distinta casuística que presentaban los solicitantes y la necesidad de un tratamiento individualizado.

Los solicitantes tienen dificultades a la hora de realizar y poner sobre el papel los análisis necesarios sobre su desempeño profesional. Nos encontramos que las autoevaluación realizadas carecen en algunos casos del rigor y la coherencia necesarios a la hora de establecer las necesarias relaciones entre la actividad realizada y las competencias alcanzadas. Las aportaciones de los estudiantes sobre las competencias que han obtenido a través de su práctica profesional, así como su nivel de desempeño carecen de argumentación sólida al no estar respaldadas por un proceso reflexivo.

Entendemos que este proceso, con todas sus dificultades es absolutamente necesario, no solo porque así nos lo exigen a las Universidades, sino para hacer frente a una realidad, la adquisición de competencias por la vía de la experiencia laboral, cada vez más documentada.

\section{Conclusiones y propuestas de mejora}

Sin duda, la experiencia en ambas convocatorias ha sido muy positiva y, aunque requiere un mayor desarrollo para lograr un procedimiento que mejore la garantía de la objetividad y validez del proceso, consideramos que responde a la necesidad surgida para reconocer diferentes vías de formación, más allá de la meramente formal. Por ello, la creación de este procedimiento, a raíz de los resultados obtenidos, avalan la propuesta y, por tanto, su continuidad. Se evidencia el importante número de profesionales que desempeñan funciones específicas como educador social sin tener esta titulación, por lo que este reconocimiento flexibiliza y favorece la adquisición de la titulación que se requiere en el ámbito laboral a un importante número de profesionales. Por otro lado, la sociedad del conocimiento exige una mayor flexibilidad en todos estos procesos, sin perder la garantía de la fiabilidad y validez de todo procedimiento evaluativo. Poder dar respuesta a estas situaciones es, tal vez, el mayor logro de esta experiencia, aunque somos conscientes de que nuestra propuesta tiene todavía muchos aspectos a mejorar. Por ello, proponemos para próximas convocatorias: 
- Elaborar un documento-guía explicando, de forma más detallada, la finalidad de este reconocimiento, quién lo puede solicitar, cuándo debe solicitarse, qué documentos se requieren, cómo se realiza una autoevaluación motivada, etc.

- Proponer una convocatoria anual con numerus clausus y con un cronograma concreto, contando con el apoyo del COIE. Es absolutamente necesario lograr un proceso coordinado entre una comisión académica, el personal administrativo responsable de los reconocimientos y el COIE.

- Realizar entrevistas por competencias a todos los estudiantes solicitantes del reconocimiento, ya que garantiza la validez y fiabilidad del proceso y es el mejor escenario para contrastar la realidad de estos aprendizajes, apoyándonos en su capacidad de autoevaluación.

- Valorar qué asignaturas son susceptibles de reconocimiento por esta vía, y cuáles no, ya que no debemos perder de vista la dimensión formativa que entraña toda titulación universitaria. Las materias con mayor carga teórica aportan a los estudiantes una formación clave en su desarrollo profesional, por lo que hay que valorar en qué situaciones pueden ser objeto de reconocimiento.

- Recomendar a los estudiantes que este procedimiento debe solicitarse a partir del segundo curso de la titulación, una vez posean un conocimiento básico de las competencias y contenidos del título. Nunca debe convertirse en un procedimiento meramente burocrático.

- No descartamos la posibilidad de la observación en el puesto de trabajo, en la que se contaría con la colaboración de los profesores tutores de los Centros Asociados de la UNED, dada la dispersión geográfica de nuestros estudiantes.

Quedan muchos ámbitos por abordar, como es la experiencia adquirida a través del voluntariado, pero consideramos que lo idóneo es fundamentar correctamente una vía de aprendizaje, antes de iniciar otras. De todas formas, iniciar este procedimiento ha supuesto ya una respuesta a las demandas de flexibilidad y empleabilidad de los profesionales de este siglo XXI y a reconocer de forma efectiva la sociedad del conocimiento en la que estamos inmersos como la sociedad del aprendizaje que debe ser.

\section{Referencias bibliográficas}

CEDEFOP (2013a). On the way to 2020: data for vocational education and training policies. Luxembourg: Publications Office of the European Union. Recuperado de: http://www.cedefop.eurooa.eu/EN/Files/5533_en.pdf, consultado 12 de Julio de 2013.

CEDEFOP (2013b). Benefits of vocational education and training in Europe for people, organizations and countries. Luxembourg: Publications Office of the European Union. Recuperado de: http://www.cedefop.europa.eu/EN/Files/4121_en.pdf, consultado 12 de Julio de 2013. 
COM (2012a). Council Recommedation of 20 December 2012 on the validation of no formal and informal learning. 398/01. Recuperado de: http://eurlex.europa.eu/LexUriServ/LexUriServ.do?uri=OJ:C2012:398:0001:0005:EN:PDF, consultado 20 de febrero de 2013.

COM (2012b). Un nuevo concepto de educación: invertir en las competencias para lograr mejores resultados socioeconómicos. 669. Recuperado de: http://ec.europa.eu/education/news/rethinking/com669_es.pdf, consultado 20 de septiembre de 2012.

COM (2012c). Recomendación del Consejo sobre la validación del aprendizaje no formal e informal. 485 Recuperado de: http://ec.europa.eu/education/lifelonglearning-policy/doc/informal/proposal2012_es.pdf, consultado 20 de septiembre de 2012.

EUROPEAN COMMISSION; CEDEFOP; ICF International (2014). European inventory on validation of non-formal and informal learning 2014: country report Spain. Recuperado de: http://libserver.cedefop.europa.eu/vetelib/2014/87056_ES.pdf, consultado 4 de febrero de 2015.

FOREM (2010). Estudio comparativo de los modelos europeos de acreditación de competencias. Madrid: Fundación Tripartita Recuperado de: http://www.fundaciontripartita.org/almacenv/webpubpro/textbase/aacc02/anexos/20 100178.pdf, consultado 20 febrero de 2013.

LEY ORGÁNICA 4/2007, de 12 de abril, por la que se modifica la Ley Orgánica 6/2001, de 21 de diciembre, de Universidades. Boletín Oficial del Estado, de 13 de abril de 2007, núm. 89, pp. 16241-16260. Recuperado de: http://www.boe.es/buscar/doc.php?id=BOE-A-2007-7786, consultado 15 de marzo de 2011.

LEY ORGÁNICA 5/2002, de 19 de junio, de las Cualificaciones y de la Formación Profesional. Boletín Oficial del Estado, de 20 de junio de 2002, núm. 147, pp. 22437-22442. Recuperado de: http://www.boe.es/buscar/doc.php?id=BOE-A2002-12018, consultado 15 de marzo de 2011.

MANZANO, N.; MARTÍN CUADRADO, A. y RUIZ-CORBELLA, M. (2012). El reconocimiento de las competencias profesionales adquiridas por experiencia laboral: la formación de orientadores, asesores y evaluadores. Revista de Educación, 359, 657-673. doi: 10.4438/1988-592X-RE-2012-359-203.

MEDINA, O. Y SANZ FERNÁNDEZ, F. (2009a). Los sistemas de reconocimiento y acreditación de los aprendizajes formales e informales: referencias históricas, funciones socioeducativas y perspectiva teórica. Revista de Educación, 348, 253281.

MEDINA, O. Y SANZ FERNÁNDEZ, F. (2009b). El reconocimiento y la acreditación de la experiencia. Teoría de la Educación. Revista Interuniversitaria, 22, 165-193. 
NUFFIC (coord) (2012). European Area of Recognition Manual. Recuperado de: http://www.eurorecognition.eu/manual/ear_manual_v_1.0.pdf, consultado 20 de febrero de 2013

NUFFIC et al. (2009). Recognition of Non-formal and Informal Learning: Learning Outcomes. Final report, The Hague. Recuperado de:

REAL DECRETO 1128/2003, de 5 de septiembre, por el que se regula el Catálogo Nacional de Cualificaciones Profesionales. Boletín Oficial del Estado, de 17 de septiembre de 2003, núm. 223, pp. 34293-34296. Recuperado de: https://www.boe.es/diario_boe/txt.php?id=BOE-A-2003-17588, consultado 15 de marzo de 2011.

REAL DECRETO 1224/2009, de 17 de julio, reconocimiento de las competencias profesionales adquiridas por experiencia laboral. Boletín Oficial del Estado, de 25 de agosto de 2009, núm. 205, pp. 72704-72727. Recuperado de: https://www.boe.es/buscar/doc.php?id=BOE-A-2009-13781, consultado 15 de marzo de 2011.

REAL DECRETO 861/2010 (2010), de 2 de julio, por el que se modifica el Real Decreto 1393/2007, de 29 de octubre, por el que se establece la ordenación de las enseñanzas universitarias oficiales. Boletín Oficial del Estado, 3 de julio de 2010, núm. 161, pp. 58454-58468. Recuperado de: http://www.boe.es/buscar/doc.php?id=BOE-A-2010-10542, consultado 15 de marzo de 2011.

RETORTILLO, A. (2011). La evaluación, reconocimiento y acreditación de los aprendizajes no formales e informales en el ámbito universitario: elementos para el debate. Revista Electrónica Interuniversitaria de Formación del Profesorado, 14 (1), 218-226.

RIMBAU, E., ARMAYONES, M., DELGADO, A., MAS, X. Y RIFÀ, H. (2008). El reconocimiento de los aprendizajes adquiridos por la experiencia previa: un nuevo reto para el sistema universitario. Estudios sobre Educación, 15, 31-51.

SANZ FERNÁNDEZ, F. (2005). La validación universitaria de los aprendizajes adquiridos en la experiencia: su contexto económico y social. Revista de Educación, 338, 101-124.

SOBRADO, L. (2003). Evaluación y acreditación de cualificaciones profesionales. Bordón. Revista de Pedagogía, 55 (3), 365-378.

TEJADA, J. (2011). La evaluación de las competencias en contextos no formales: dispositivos e instrumentos de evaluación. Revista de Educación, 354, 731-745, doi: 10.4438/1988-592X-RE-2011-354-018.

TEJADA, J. (2012). La alternancia de contextos para la adquisición de competencias profesionales en escenarios complementarios de educación superior: marco y estrategia. Educación XX1, 15 (2), 17 - 40. 
WERQUIN, P. (2010). Recognition of Non-Formal and Informal Learning: Country Practices. Paris: OECD. Recuperado de: http://www.oecd.org/education/skillsbeyond-school/44600408.pdf, consultado 20 de febrero de 2013.

\section{Correspondencia con los autores}

Miriam GARCÍA BLANCO

Dpto Teoría de la Educación y Pedagogía Social.

Facultad de Educación

Universidad Nacional de Educación a Distancia (UNED)

C/ Juan del Rosal, 14

28040 Madrid (España)

e-mail: mgblanco@edu.uned.es

María José BAUTISTA-CERRO

Dpto Teoría de la Educación y Pedagogía Social.

Facultad de Educación

Universidad Nacional de Educación a Distancia (UNED)

C/ Juan del Rosal, 14

28040 Madrid (España)

e-mail:mjbautistac@edu.uned.es

Marta RUIZ CORBELLA

Dpto Teoría de la Educación y Pedagogía Social.

Facultad de Educación

Universidad Nacional de Educación a Distancia (UNED)

C/ Juan del Rosal, 14

28040 Madrid (España)

e-mail: mruiz@edu.uned.es 